\title{
Inter-comparison of spatial upscaling methods for evaluation of satellite-based soil moisture
}

\author{
Jun Qin ${ }^{\mathrm{a}, *}$, Long Zhao ${ }^{\mathrm{a}}$, Yingying Chen ${ }^{\mathrm{a}}$, Kun Yang ${ }^{\mathrm{a}}$, Yaping Yang ${ }^{\mathrm{b}}$, Zhuoqi Chen ${ }^{\mathrm{c}}$, Hui Lu ${ }^{\mathrm{d}}$

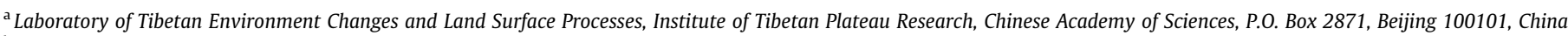 \\ ${ }^{\mathrm{b}}$ State Key Laboratory of Resources and Environmental information, Institute of Geographic Sciences and Natural Resources Research, Chinese Academy of Science, Beijing \\ 100101, China \\ ${ }^{\mathrm{c}}$ College of Global and Earth System Science, Beijing Normal University, Beijing 100875, China \\ ${ }^{\mathrm{d}}$ Ministry of Education Key Laboratory for Earth System Modeling, Center for Earth System Science, Tsinghua University, Beijing 100084, China
}

\section{A R T I C L E I N F O}

\section{Article history:}

Received 5 August 2014

Received in revised form 17 November 2014

Accepted 23 January 2015

Available online 4 February 2015

This manuscript was handled by

Konstantine P. Georgakakos, Editor-in-Chief,

with the assistance of Matthew McCabe,

Associate Editor

\section{Keywords:}

Satellite

Soil moisture

Evaluation

Upscaling

MODIS

\begin{abstract}
S U M M A R Y
Soil moisture is a key factor in energy and water cycles. Many satellite missions have been planned and implemented for retrieving soil moisture globally. Because the spatial representativeness of a point-scale soil moisture station is rather limited, a station network needs setting up for scale-matching validation of satellite-based soil moisture products. Even so, an upscaling procedure is needed to upscale these station soil moisture values into area-wide one. However, such a procedure itself introduces uncertainties into the upscaled soil moisture. In this study, four upscaling methods (simple average, block kriging, model-based, and apparent-thermal-inertia-based) are inter-compared according to their performance stability for evaluation of soil moisture estimated by assimilating microwave signals into a land surface model. It is found that the performance of the model-based upscaling approach is the most unstable because model simulations are full of uncertainties for representing spatial variability of soil moisture. The block kriging upscaling method performs not worse than the simple averaging approach; the former may generate more representative soil moisture if the range of the soil moisture semivariogram used in the block kriging is comparable to the extent of a satellite footprint. The apparent-thermal-inertia-based upscaling is the most stable one, which has been developed with the aid of high-resolution satellite thermal data. All analyses indicate that choosing a suitable upscaling approach is important for the effective evaluation of satellite-based soil moisture. Otherwise, uncertainties hiding in the upscaling method will be incorrectly attributed to errors in satellite products, undermining our confidence in implementing them into practice.
\end{abstract}

(c) 2015 Elsevier B.V. All rights reserved.

\section{Introduction}

It is widely recognized that the acquisition of the spatiotemporal distribution of soil moisture at regional and global scales is of importance because it controls the exchanges of energy, water, and carbon between the land surface and the atmosphere. A deep understanding of these processes improves our skills in modeling climate and land hydrology (Hirabayashi et al., 2005; Koster et al., 2010; Seneviratne et al., 2010; Sheffield and Wood, 2007). Therefore, a number of microwave satellite missions have been initiated to map land surface moisture, such as the demised Advanced Microwave Scanning Radiometer for EOS (AMSR-E) (Njoku et al., 2003), the ongoing Soil Moisture and Ocean Salinity (SMOS) mission (Kerr et al., 2010), and the upcoming Soil Moisture Active

\footnotetext{
* Corresponding author.

E-mail address: shuairenqin@gmail.com (J. Qin).
}

Passive (SMAP) mission (Entekhabi et al., 2010a). There are generally two methods to estimate soil moisture based on microwave signals. One is the inversion of a radiative transfer model based upon observed brightness temperatures with some ground ancillary information (Das et al., 2011; Jackson et al., 1999; Kerr et al., 2012). The other is the assimilation of brightness temperatures into a land surface model driven by atmospheric forcing (Crow and Wood, 2003; Lu et al., 2012; Montzka et al., 2011; Pan and Wood, 2006; Qin et al., 2009; Reichle et al., 2002; Yang et al., 2007). No matter which approach is used, soil moisture estimates from satellites have to be evaluated against in-situ measurements before applied in practice.

It is well known that in-situ soil moisture is merely representative over a small spatial scale (Bloschl and Grayson, 2001) because of its high spatial variability caused by spatial heterogeneity of soil, vegetation, topography, and precipitation. On the other hand, the spatial scale of satellite footprints is much larger than that of 
in-situ soil moisture measurements. This scale-mismatch has already been noticed in field calibration/validation campaigns for satellite soil moisture products (Al Bitar et al., 2012; Jackson et al., 2012, 2010; Sanchez et al., 2012). In order to eliminate such a mismatch, several spatial upscaling methods have been proposed to convert point-scale in-situ soil moisture to footprint-scale one.

There are generally four commonly used upscaling methods based on a station network (Crow et al., 2012; Qin et al., 2013). The first approach, which is the most often used, is the simple averaging of station data. The second approach is the block kriging, which utilizes the spatial correlation structure (semivariogram) of soil moisture measurements among stations to calculate areawide soil moisture. The third approach is model-based, which uses the spatial pattern of soil moisture simulated by a land surface model to perform upscaling. The fourth approach is apparent-thermal-inertia-based, which merges in-situ data with soil moisture derived from fine-resolution satellite thermal signals.

As a matter of fact, it is very important to select a suitable upscaling approach to evaluating satellite-based soil moisture estimates. Otherwise, uncertainties intrinsic to the upscaling approach will be brought into the upscaled soil moisture, leading to biased evaluations. Therefore, the upscaling approaches themselves need to be carefully examined. In this study, we inter-compare the four upscaling methods by using their upscaled soil moisture to evaluate soil moisture estimated by microwave data assimilation on the Tibetan Plateau (TP) and the Mongolian Plateau (MP), and analyze the cause of different performances of the four upscaling methods.

\section{Study area and data}

Two soil moisture measuring networks deployed on the Tibetan Plateau and the Mongolian Plateau are used in this study. The Tibetan Plateau Soil Moisture/Temperature Monitoring Network (SMTMN) is located in the central TP and set up around the town of Naqu within an area of $\sim 100 \mathrm{~km} \times 100 \mathrm{~km}$ over $4500 \mathrm{~m}$ above sea level. The surface is relatively flat in most of this area although rugged in some places. The land cover is primarily alpine meadow with a few wetlands scattering. The SMTMN was initiated in 2010. Thirty-nine stations were first installed along four roads (white lines in Fig. 1a). In 2011, twenty more stations were installed. Some of them were used to replace the lost and damaged ones installed in 2010 and the others in conjunction with part of original stations form a medium network (green box in Fig. 1a) with a spatial size of $25 \mathrm{~km} \times 25 \mathrm{~km}$ nested in the large one (red box in Fig. 1a). Five more stations were installed in a $5 \mathrm{~km} \times 5 \mathrm{~km}$ area in 2012 . These five new stations and nearby four stations compose a small network (blue box in Fig. 1a). At each station, both soil moisture and temperature are measured at depths of $0-5,10,20$, and $40 \mathrm{~cm}$, respectively, and the sampling interval is set to be $30 \mathrm{~min}$. In this study, only surface soil moisture data that are measured at 28 stations in the large network (marked as red solid circles in Fig. 1a) from June 1 to September 302011 are used over the TP. The readers are referred to the article by Yang et al. (2013) for more information on this network.

The other network is located at Mandalgobi of Mongolia, which is a reference site of the Coordinated Enhanced Observing Period program (Kaihotsu et al., 2005). It covers a flat area of $120 \mathrm{~km} \times 160 \mathrm{~km}$ with a mean elevation of $1380 \mathrm{~m}$ above sea level. The land cover type is primarily grassland. In this network, 12 Automatic Stations for Soil Hydrology (ASSH) and 6 Automatic Weather Stations (AWS) were deployed. Their geographic locations are shown in Fig. 1b. At ASSH, soil temperature and moisture are measured at depths of 3 and $10 \mathrm{~cm}$ and the sampling interval is $30 \mathrm{~min}$. The AWS measure both meteorological data (wind, temperature, humidity, pressure, precipitation, and net radiation) and soil moisture/temperature profiles. Only surface soil moisture data from May 1 to September 232003 are used. Considering data continuity, we only use 14 stations marked as red solid circles in Fig. $1 b$.

\section{Methodologies}

In this study, the following way is taken to inter-compare and analyze these upscaling methods. First, an assimilation algorithm is applied to assimilate AMSR-E brightness temperatures or SMOS soil moisture products into a land surface model to obtain surface moisture estimates in the two networks. Second, the four upscaling approaches are implemented to upscale in-situ soil moisture to obtain area-wide values in the two network areas, respectively. Third, the upscaled soil moisture values are used to evaluate the assimilation results. Finally, the cause that leads to their different performances is investigated. In the following, the upscaling approaches and the assimilation system are briefly introduced.

\subsection{Upscaling approaches}

The aim of any upscaling method can be mathematically abstracted as:

$$
\bar{\theta}_{t}^{\text {ups }}=U\left(\boldsymbol{\theta}_{t}^{\mathrm{obs}}\right),
$$
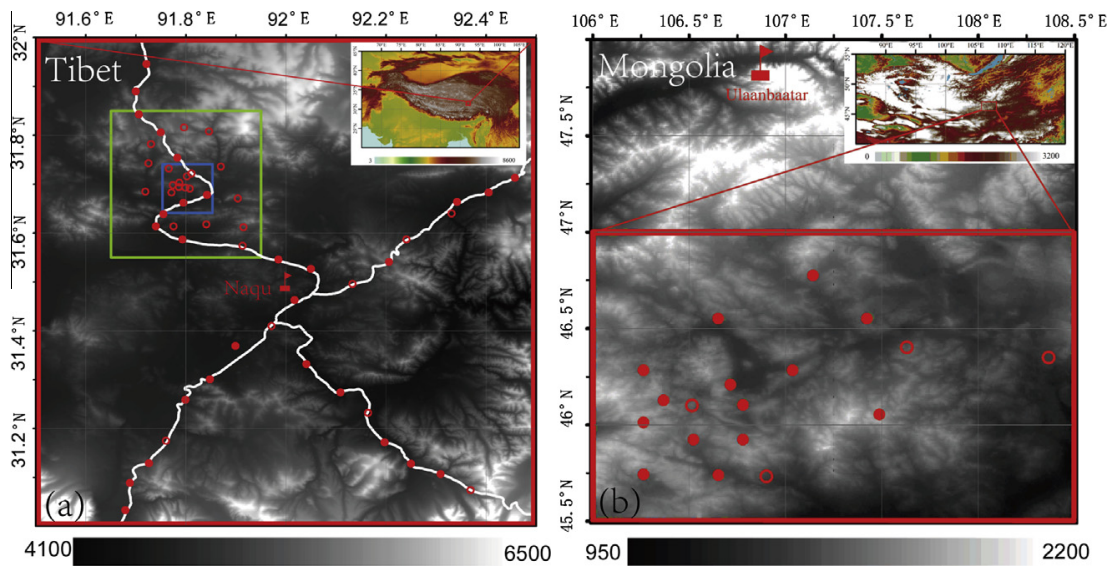

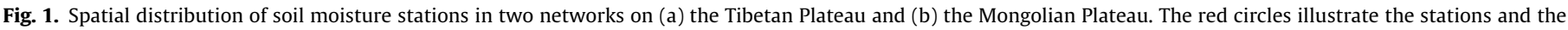
filled circles mark those used in this study. (For interpretation of the references to colour in this figure legend, the reader is referred to the web version of this article.) 
with

$\boldsymbol{\theta}_{t}^{\mathrm{obs}}=\left[\theta_{t, 1}^{\mathrm{obs}}, \theta_{t, 2}^{\mathrm{obs}}, \ldots, \theta_{t, N}^{\mathrm{obs}}\right]^{T}$,

where $U(\cdot)$ denotes the upscaling function, $\bar{\theta}_{t}^{\text {ups }}\left(\mathrm{m}^{3} \mathrm{~m}^{-3}\right)$ the upscaled soil moisture in an area, $\theta^{\text {obs }}\left(\mathrm{m}^{3} \mathrm{~m}^{-3}\right)$ the in-situ soil moisture measurement, $N$ the number of stations, and $t$ the recording time. The core of the upscaling process is to identify the concrete form of $U(\cdot)$ with the aid of extra information. The four methods adopt a linear functional form and thus Eq. (1) can be expressed as:

$\bar{\theta}_{t}^{\text {ups }}=\left(\boldsymbol{\theta}_{t}^{\text {obs }}\right)^{T} \boldsymbol{\beta}$,

where $\boldsymbol{\beta}$ denotes the regression coefficient vector to be found according to an upscaling method. So, the upscaling process essentially becomes the determination of $\boldsymbol{\beta}$.

The first method is the simple averaging (hereafter referred to as SA), which can be formulated as:

$\bar{\theta}_{t}^{\text {ups }}=\frac{1}{N} \sum_{i=1}^{N} \theta_{t, i}^{\mathrm{obs}}$.

After some simple algebraic manipulations, the regression coefficient vector of the simple averaging can be expressed as $\boldsymbol{\beta}_{\mathrm{SA}}=[1 /$ $N, \ldots, 1 / N]^{T}$ if Eq. (4) is expressed in the matrix form of Eq. (3). The extra information used in this approach is the assumption that the simple arithmetic mean at a limited number of stations could represent the true area-wide soil moisture.

The second upscaling method is the block kriging (Vinnikov et al., 1999; hereafter referred to as BK), which can be expressed as:

$\bar{\theta}_{t}^{\text {ups }}=\left(\boldsymbol{\theta}_{t}^{\text {obs }}\right)^{T} \mathbf{C}^{-1} \mathbf{D}$,

where $\boldsymbol{C}$ is an $N \times N$ matrix containing the covariance values among the components of $\boldsymbol{\theta}_{t}^{\mathrm{obs}}$, and $\boldsymbol{D}$ is an $N \times 1$ vector containing the covariance values between the components of $\theta_{t}^{\text {obs }}$ and the true area-wide moisture values. It is clear that the regression coefficient vector of the block kriging can be expressed as $\boldsymbol{\beta}_{\mathrm{BK}}=\boldsymbol{C}^{-1} \boldsymbol{D}$. The extra information used in this method is the spatial correlation hiding in the soil moisture measurements in an area. This correlation can be represented as either covariance or semivariogram and estimated based on in-situ soil moisture at different stations.

The third one is the model-based approach (Crow et al., 2005; hereafter referred to as MB). This approach needs to exploit finegrid soil moisture simulated by a land surface model in a network area. It can be formulated as:

$\bar{\theta}_{t}^{\mathrm{ups}}=\bar{\theta}_{t}^{\mathrm{sim}}+\frac{1}{N} \sum_{i=1}^{N}\left(\theta_{t, i}^{\mathrm{obs}}-\theta_{t, i}^{\mathrm{sim}}\right)$,

with

$\bar{\theta}_{t}^{\text {sim }}=\frac{1}{M} \sum_{j=1}^{M} \theta_{t, j}^{\text {sim }}$

where $\theta_{t, j}^{\mathrm{sim}}\left(\mathrm{m}^{3} \mathrm{~m}^{-3}\right)$ denotes the simulated soil moisture at grid box $j$ in an area, $\bar{\theta}_{t}^{\text {sim }}\left(\mathrm{m}^{3} \mathrm{~m}^{-3}\right)$ the averaged soil moisture simulations over all grid boxes, and $\theta_{t, i}^{\text {sim }}\left(\mathrm{m}^{3} \mathrm{~m}^{-3}\right)$ the simulated soil moisture in an grid box enclosing the moisture station $i$, and $M$ the number of grid boxes in an area. If an augmented form of $\boldsymbol{\theta}_{t}^{\text {obs }}$ is taken as follows:

$\tilde{\boldsymbol{\theta}}_{t}^{\mathrm{obs}}=\left[\left(\bar{\theta}_{t}^{\mathrm{sim}}-\theta_{t, 1}^{\mathrm{sim}}\right),\left(\bar{\theta}_{t}^{\mathrm{sim}}-\theta_{t, 2}^{\mathrm{sim}}\right), \ldots,\left(\bar{\theta}_{t}^{\mathrm{sim}}-\theta_{t, N}^{\mathrm{sim}}\right), \theta_{t, 1}^{\mathrm{obs}}, \theta_{t, 2}^{\mathrm{obs}}, \ldots, \theta_{t, N}^{\mathrm{obs}}\right]^{T}$,

then the upscaling function similar to Eq. (3) can be expressed as:

$\bar{\theta}_{t}^{\text {ups }}=\left(\tilde{\boldsymbol{\theta}}_{t}^{\mathrm{obs}}\right)^{T} \tilde{\boldsymbol{\beta}}_{\mathrm{MB}}$ with

$\tilde{\boldsymbol{\beta}}_{\mathrm{MB}}=[1 / N, 1 / N, \ldots, 1 / N]^{T}$.

The extra information for this approach is the assumption that the differences between simulated and measured soil moisture values are less spatially variable than in-situ measurements, and thus can be more reliably upscaled.

As shown in many studies (Merlin et al., 2008; Piles et al., 2011), satellite thermal data are closely related to soil moisture. The fourth method uses MODIS-derived apparent thermal inertia (ATI) as the extra information to find the regression coefficient vector $\tilde{\boldsymbol{\beta}}_{\text {ATI }}$ (Qin et al., 2013). This information is not used in the other three upscaling methods. Its basic idea is to minimize the following cost function $(J)$ with respect to $\tilde{\beta}_{\text {ATI }}$ by Bayesian linear regression:

$J=\sum_{t=1}^{T}\left[\bar{\theta}_{t}^{\mathrm{ati}}-\left(\tilde{\boldsymbol{\theta}}_{t}^{\mathrm{obs}}\right)^{T} \tilde{\beta}_{\mathrm{ATI}}\right] \sigma^{-2}\left[\bar{\theta}_{t}^{\mathrm{ati}}-\left(\tilde{\boldsymbol{\theta}}_{t}^{\mathrm{obs}}\right)^{T} \tilde{\beta}_{\mathrm{ATI}}\right]+\alpha \tilde{\beta}_{\mathrm{ATI}}^{T} \tilde{\beta}_{\mathrm{ATI}}$

with

$\tilde{\boldsymbol{\theta}}_{t}^{\mathrm{obs}}=\left[1, \theta_{t, 1}^{\mathrm{obs}}, \theta_{t, 2}^{\mathrm{obs}}, \ldots, \theta_{t, N}^{\mathrm{obs}}\right]^{T}$

and

$\bar{\theta}_{t}^{\mathrm{ati}}=g\left(\bar{\tau}_{t}\right)$,

where $\bar{\theta}_{t}^{\text {ati }}\left(\mathrm{m}^{3} \mathrm{~m}^{-3}\right)$ denotes the representative ATI-derived mean soil moisture in an area of concern; $\bar{\tau}_{t}$ the pixel-averaged ATI value in this area; $g(\cdot)$ the functional relationship between soil moisture and ATI derived by empirically regressing station-averaged soil moisture against corresponding pixel-averaged ATI values; $\sigma\left(\mathrm{m}^{3} \mathrm{~m}^{-3}\right)$ the standard deviation of $\bar{\theta}_{t}^{\text {ati }} ; \alpha(-)$ the regularization parameter to avoid overfitting; and $T$ the number of dates when the pixel-averaged ATI is available. ATI is defined as:

$\tau=C \frac{1-a}{A}$

where $\tau\left(K^{-1}\right)$ denotes ATI, $C(-)$ the correction factor to account for seasonal variation of solar radiation, $a(-)$ the surface albedo, and $A$ $(K)$ the amplitude of diurnal land surface temperature (LST) cycle, which can be derived from the MODIS LST product. Readers are referred to the article by Qin et al. (2013) for more details on this ATI-based upscaling method.

\subsection{Assimilation framework}

The assimilation framework developed by Yang et al. (2007) is used to assimilate satellite observations into a simple biosphere model (SiB2) (Sellers et al., 1996) on the TP and the MP. Its most distinctive feature is the ability to estimate both model parameters and soil moisture. This is achieved by performing optimization within a dual-pass framework. In the first pass, one cost function is constructed to measure the differences between simulated brightness temperatures via a radiative transfer model and corresponding satellite observed ones (or between model simulated soil moisture values and satellite retrieved ones) during a long-term window and then is minimized with respect to model parameters (soil texture and porosity) that are identified to strongly impact moisture simulation. The first pass is only conducted once. In the second pass, another cost function is constructed to measure the difference between simulated brightness temperature and observed one (or between modeled soil moisture and retrieved one) within a short-term window and then minimized with respect to soil moisture. The second pass is generally conducted at a daily time scale. The readers can refer to the appendix for more details of this assimilation framework. 
In this study, two types of satellite signals are assimilated. One is the brightness temperatures of AMSR-E $6.9 \mathrm{GHz}$ and $18.7 \mathrm{GHz}$ vertical polarization for both the TP and the MP. The other is the SMOS level-2 surface soil moisture product. But the latter is only taken for the TP, because the SMOS was launched in 2009 while the soil moisture network data on the MP have been unavailable since then. It is notable that the impact of radio frequency interference on SMOS is not serious over the TP although it is heavy in other regions of China. When AMSR-E brightness temperatures are assimilated, a microwave radiative transfer equation is used to convert simulated soil moisture values to microwave brightness temperatures, and then compare the brightness temperatures with satellite observations in the cost function. When the SMOS soil moisture values are assimilated, they are directly compared with model-simulated ones. The readers are referred to Zhao et al. (2014) for the SMOS data assimilation.

To drive this assimilation system, the Global Land Data Assimilation System (GLDAS) 0.25-degree meteorological forcing data (Rodell et al., 2004) are used in the MP study area. On the other hand, a newly developed high-resolution atmospheric forcing data set for China (Chen et al., 2011) is used in the TP study area.

\section{Results and discussion}

The land assimilation system is run at a time step of half an hour and the satellite signal (AMSR-E brightness temperature or SMOS soil moisture) is assimilated into the SiB2 model once it is available. However, the daily averaged soil moisture, which is computed from 48 values derived from assimilating the satellite signal in one day, are utilized for evaluation in order to reduce the effect of uncertainties in the atmospheric forcing. In this study, two performance metrics proposed by Entekhabi et al. (2010b) are used for evaluation. One is the root mean squared difference (RMSD), which measures the total degree of mismatch between estimated soil moisture and upscaled one, and the other is the unbiased root mean squared difference (ubRMSD), which measures the ability of estimates to capture the temporal variability of upscaled soil moisture. They are defined as follows:

$\operatorname{RMSD}=\sqrt{\frac{1}{D} \sum_{t=1}^{D}\left(\theta_{t}^{\mathrm{das}}-\bar{\theta}_{t}^{\mathrm{ups}}\right)^{2}}$

ubRMSD $=\sqrt{\mathrm{RMSD}^{2}-\mathrm{BIAS}^{2}}$,

with

$\mathrm{BIAS}=\frac{1}{D} \sum_{t=1}^{D}\left(\theta_{t}^{\mathrm{das}}-\bar{\theta}_{t}^{\mathrm{ups}}\right)$, where $D$ denotes the total number of days during the assimilation period, $\theta^{\text {das }}\left(\mathrm{m}^{3} \mathrm{~m}^{-3}\right)$ the assimilation soil moisture estimates, and $\bar{\theta}^{\text {ups }}\left(\mathrm{m}^{3} \mathrm{~m}^{-3}\right)$ the upscaled area-wide moisture.

Since the ATI upscaling approach is slightly complicated compared to the other upscaling methods, some intermediate results are displayed for understanding. As introduced in Section 3, an empirical relationship between ATI and soil moisture should be constructed in the ATI upscaling method. In this study, both MODIS daily LST products (MOD11A1) and 8-day surface albedo products (MCD43B3) are used to derive ATI values. Fig. 2a and b shows the relationships for the TP and MP network areas, respectively. As can be seen, the dynamic range of soil moisture on the TP is nearly twice that on the MP and the basic distribution patterns for the pairs of ATI versus soil moisture are different. So, two different functional forms (logarithmic and exponential) are used to fit these pairs and the fitting lines can capture the relationship between ATI and soil moisture. These fitted functions are used to generate the ATI-derived soil moisture (black circles in Fig. 3a1 and b1), which basically follows the data assimilation results (black lines) on both the TP and the MP, but some of them are systematically less than the assimilation results in the wet period and greater in the dry period on the TP. The ATI upscaling method exploits this information and thus the upscaled soil moisture values (black line) go through these black circles.

Fig. 3a1-a4 indicate the upscaling results by the four methods and both AMSR-E and SMOS assimilation results (red line and blue line, respectively) for the TP network. Fig. 3b1-b4 indicate the results for the MP network. The performance metrics for these four approaches are presented in Table 1. At the same time, two-sample $t$-test is conducted to determine the statistical significance of the differences among the four upscaled soil moisture data when they are used to evaluate the assimilation results. $P$ values for these tests are given in Table 2.

As can be seen in Fig. 3a1 and a2, if the SA upscaled soil moisture is regarded as the ground truth, the soil moisture estimated by AMSR-E assimilation for the TP network is underestimated during the wet period and overestimated during the dry course. However, both the underestimation and overestimation do not occur for the ATI upscaled soil moisture. These differences are also evident in terms of the performance metrics (Table 1 ). Both ubRMSD and $\operatorname{RMSD}\left(0.021 \mathrm{~m}^{3} \mathrm{~m}^{-3}\right.$ and $\left.0.021 \mathrm{~m}^{3} \mathrm{~m}^{-3}\right)$ between the ATI upscaled soil moisture and the AMSR-E data assimilation estimate are less than the corresponding ones $\left(0.030 \mathrm{~m}^{3} \mathrm{~m}^{-3}\right.$ and $\left.0.032 \mathrm{~m}^{3} \mathrm{~m}^{-3}\right)$ between the SA upscaled soil moisture and the AMSR-E assimilation one. In the TP network, the soil moisture estimated by assimilating the SMOS soil moisture data is also evaluated against the upscaled soil moisture. As shown in Table 1, the similar results
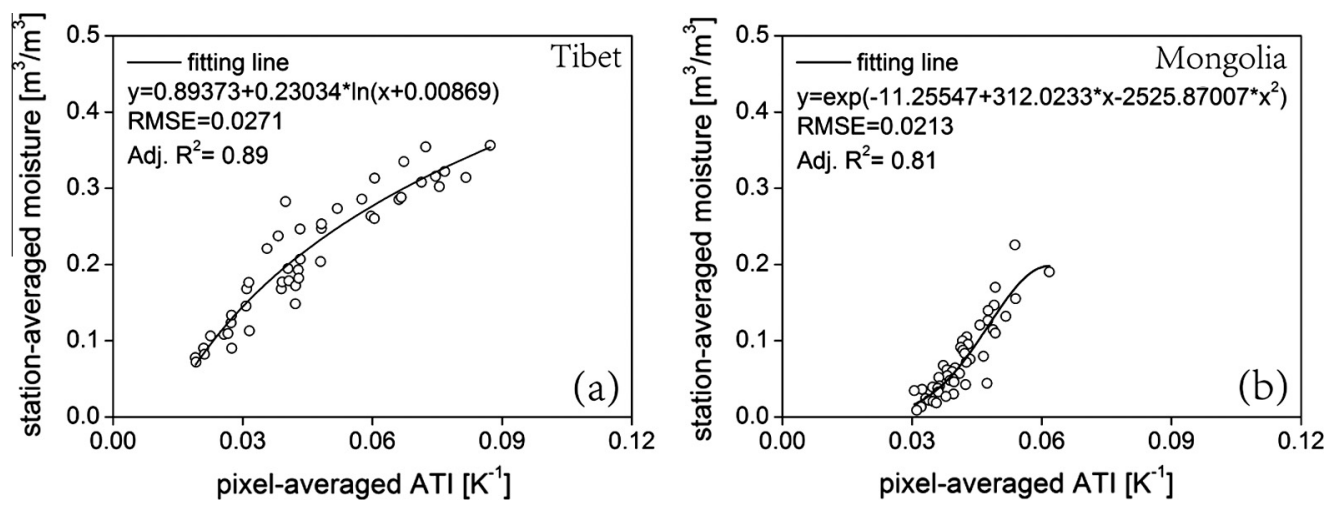

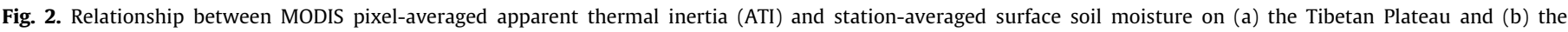
Mongolian Plateau. 


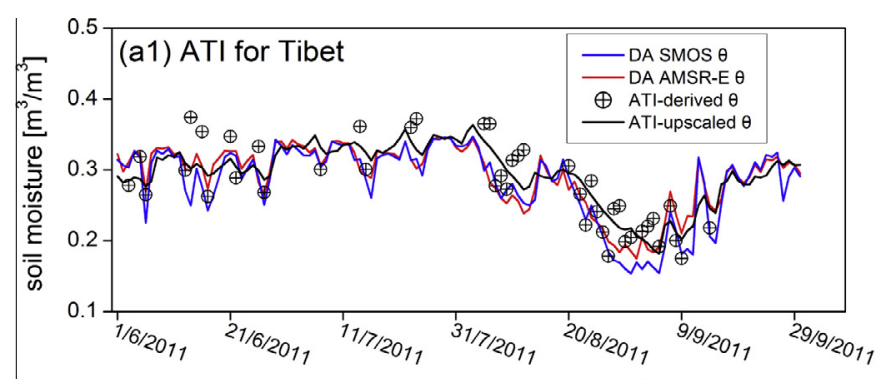

date from Jun. 1 to Sep. 30 in 2011

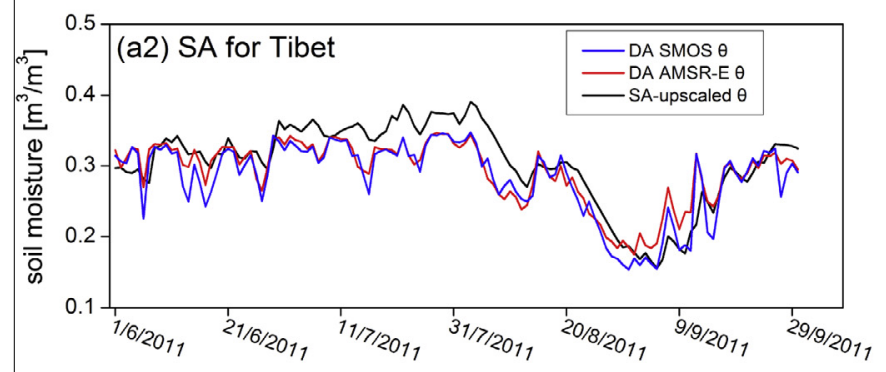

date from Jun. 1 to Sep. 30 in 2011

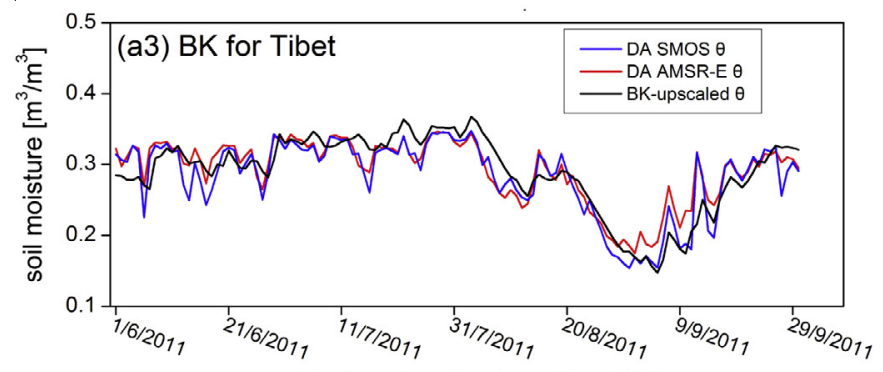

date from Jun. 1 to Sep. 30 in 2011

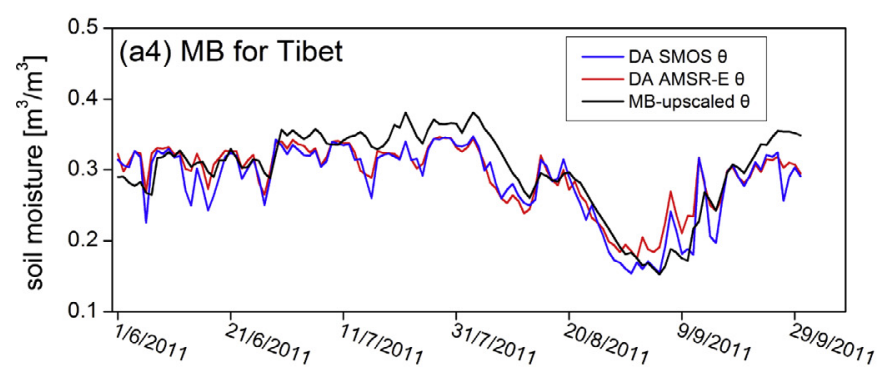

date from Jun. 1 to Sep. 30 in 2011
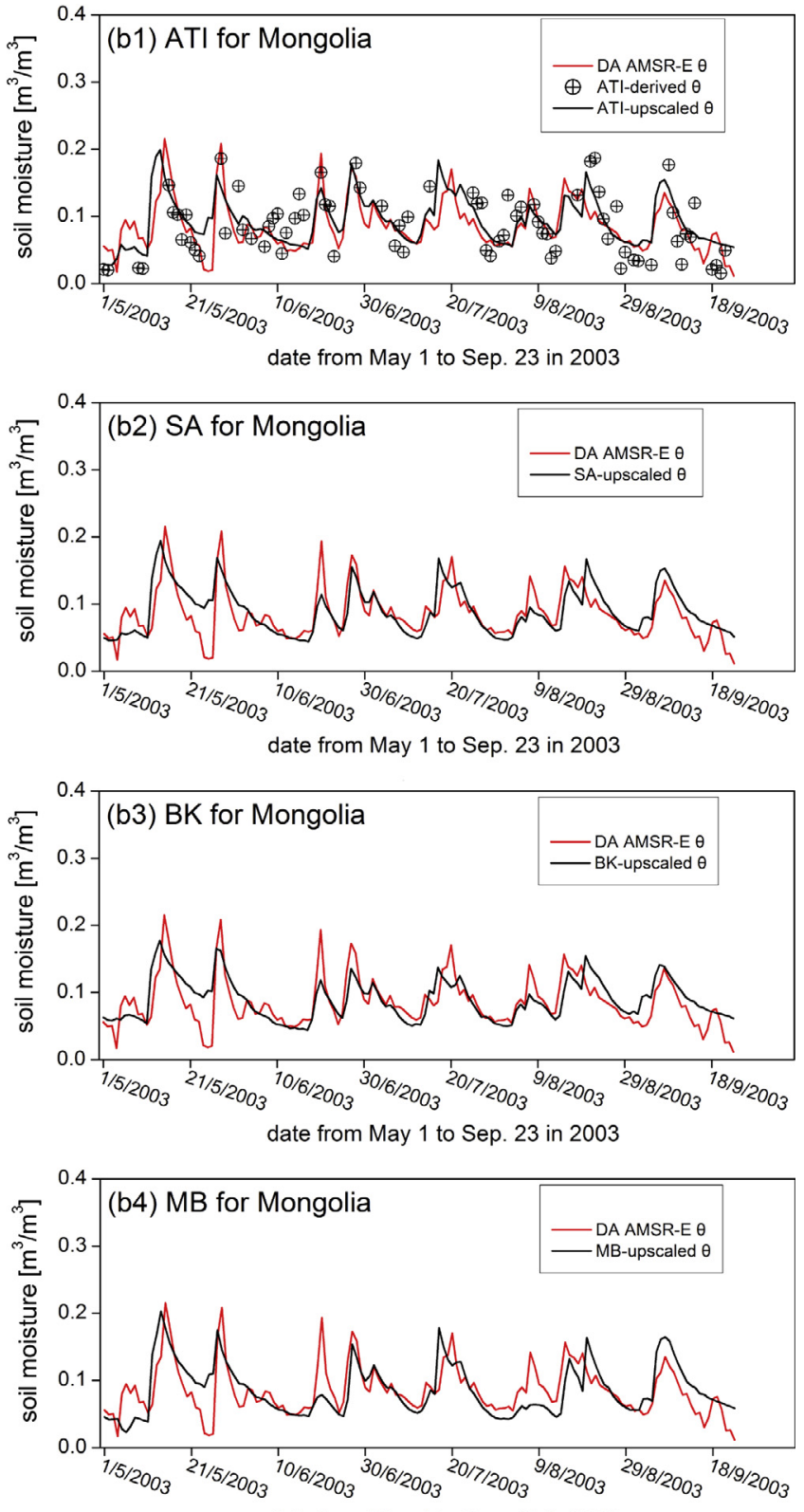

date from May 1 to Sep. 23 in 2003

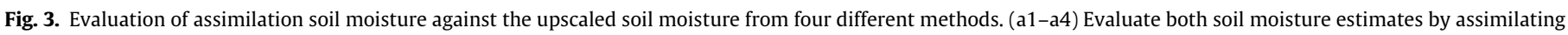

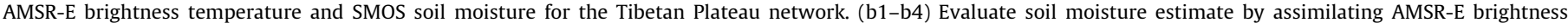

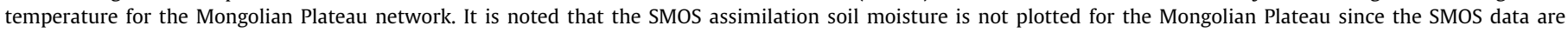
unavailable in 2003.

Table 1

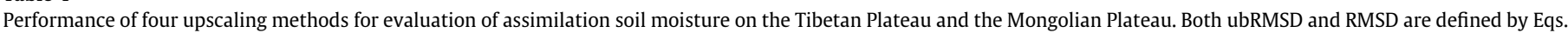
$15-17$ in units of $\mathrm{m}^{3} \mathrm{~m}^{-3}$.

\begin{tabular}{|c|c|c|c|c|c|c|c|c|c|c|c|c|}
\hline & \multicolumn{8}{|l|}{ Tibet } & \multirow{2}{*}{\multicolumn{4}{|c|}{$\begin{array}{l}\text { Mongolia } \\
\text { AMSR-E }\end{array}$}} \\
\hline & \multicolumn{4}{|c|}{ AMSR-E } & \multicolumn{4}{|l|}{ SMOS } & & & & \\
\hline & SA & ATI & BK & MB & SA & ATI & BK & MB & $\mathrm{SA}$ & ATI & BK & $\mathrm{MB}$ \\
\hline ubRMSD & 0.030 & 0.021 & 0.026 & 0.030 & 0.027 & 0.023 & 0.025 & 0.021 & 0.027 & 0.025 & 0.027 & 0.033 \\
\hline RMSD & 0.032 & 0.021 & 0.026 & 0.031 & 0.034 & 0.026 & 0.026 & 0.032 & 0.027 & 0.026 & 0.027 & 0.033 \\
\hline
\end{tabular}

are obtained for the SA and ATI upscaling methods. The consistent performance of the ATI upscaling approach is due to the fact that the ATI upscaling method digests the information of the ATIderived soil moisture, as mentioned above. The difference between the two upscaling methods is significant at $P<0.05$, as shown in Table 2.

Fig. 3b1 and b2 shows that the performance of the ATI upscaling approach for the MP network is only slightly better than that of the 
Table 2

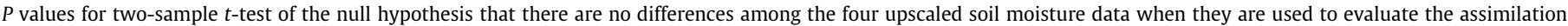
results.

\begin{tabular}{|c|c|c|c|c|c|c|c|c|c|}
\hline & \multicolumn{6}{|l|}{ Tibet } & \multicolumn{3}{|l|}{ Mongolia } \\
\hline & \multicolumn{3}{|l|}{ AMSR-E } & \multicolumn{3}{|l|}{ SMOS } & \multicolumn{3}{|l|}{ AMSR-E } \\
\hline & ATI & BK & $\mathrm{MB}$ & ATI & BK & MB & ATI & BK & MB \\
\hline SA & $1.17 \mathrm{E}-2$ & $7.23 \mathrm{E}-5$ & $3.66 \mathrm{E}-1$ & $1.13 \mathrm{E}-2$ & $2.48 \mathrm{E}-5$ & $3.20 \mathrm{E}-2$ & $2.67 \mathrm{E}-1$ & $9.80 \mathrm{E}-1$ & $2.82 \mathrm{E}-1$ \\
\hline ATI & - & $4.54 \mathrm{E}-2$ & $1.36 \mathrm{E}-2$ & - & $5.14 \mathrm{E}-2$ & $1.34 \mathrm{E}-2$ & - & $2.52 \mathrm{E}-1$ & $3.52 \mathrm{E}-2$ \\
\hline $\mathrm{BK}$ & - & -- & $2.30 \mathrm{E}-3$ & - & - & $1.20 \mathrm{E}-3$ & - & - & $2.89 \mathrm{E}-1$ \\
\hline
\end{tabular}
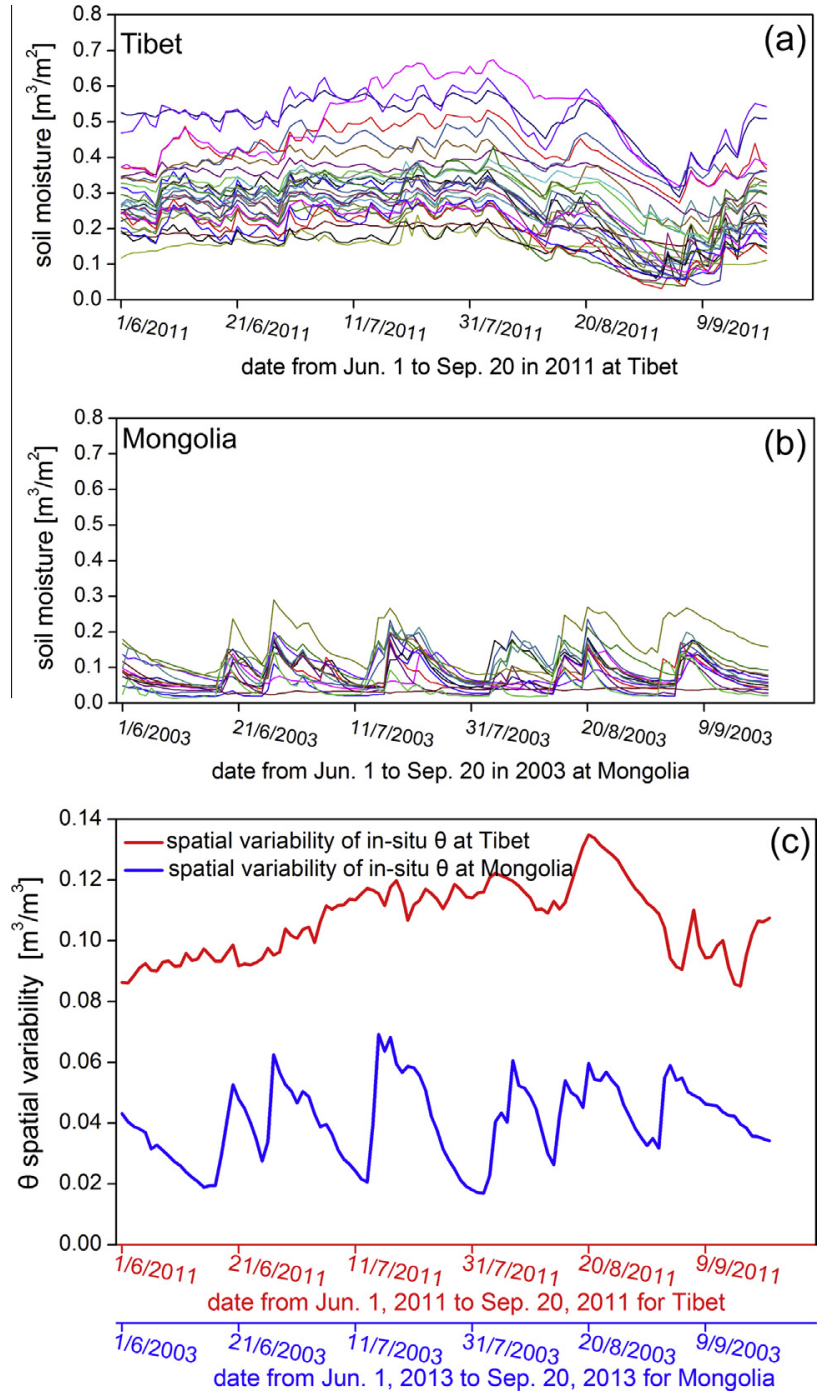

Fig. 4. Spatial variability of in-situ soil moisture. (a) Soil moisture time series observed at the stations in the Tibetan Plateau network, (b) the same as (a) but for the Mongolian Plateau network, and (c) temporal evolution of spatial variability of soil moisture on both networks.

SA upscaling in the evaluation of the AMSR-E assimilation estimate, as indicated by the values of the ubRMSD and RMSD $\left(0.027 \mathrm{~m}^{3} \mathrm{~m}^{-3}\right.$ and $0.027 \mathrm{~m}^{3} \mathrm{~m}^{-3}$ for $\mathrm{SA} ; 0.025 \mathrm{~m}^{3} \mathrm{~m}^{-3}$ and $0.026 \mathrm{~m}^{3} \mathrm{~m}^{-3}$ for ATI), and their difference is not significant, as shown in Table 2.

Herein, we investigate why the difference between the performance of the ATI and SA approaches is distinct in the two study areas. The soil moisture time series for all the stations during the dates from June 1 to September 30 but in the different years (2011 and 2003, respectively) are presented in Fig. 4a and b; and
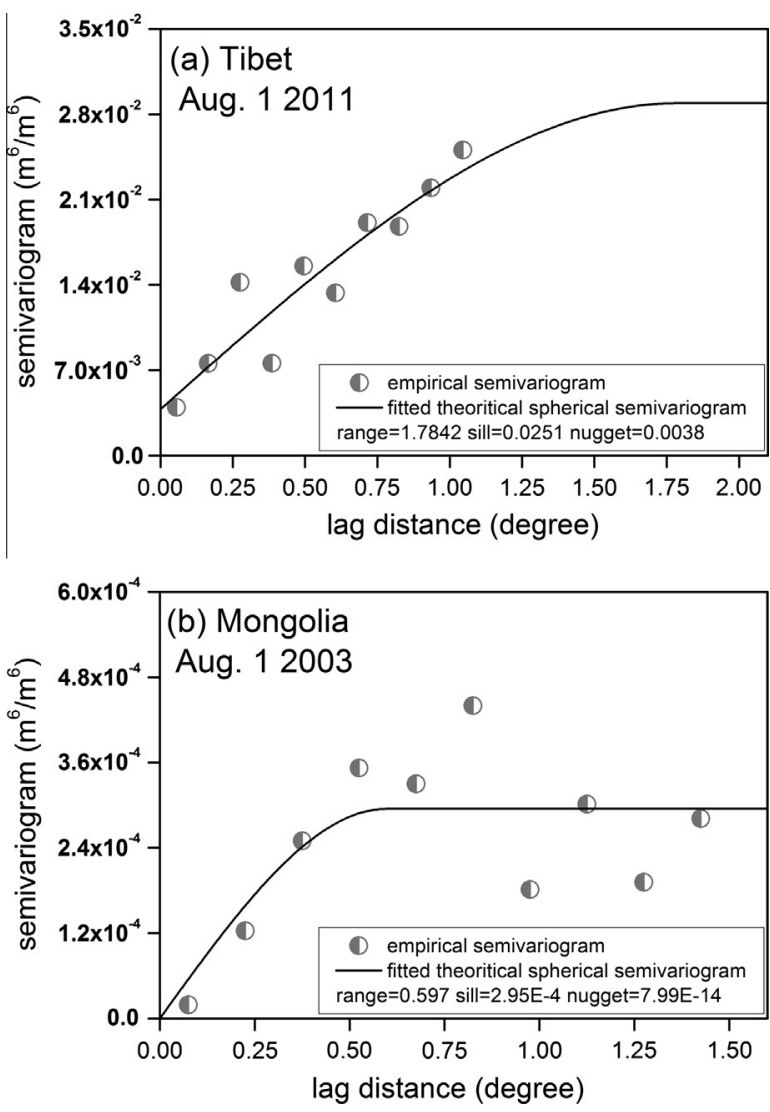

Fig. 5. Empirical and theoretical semivariograms on (a) the Tibetan Plateau on August 1, 2011 and (b) the Mongolian Plateau on August 1, 2003.

the temporal evolutions of the spatial variability over the two areas are plotted in Fig. 4c. Here, the spatial variability is defined as the standard deviation of soil moisture at all the stations. As can be seen, the magnitude of the spatial variability for the TP is nearly twice that for the MP. Therefore, the capability of using the SA upscaled soil moisture to capture the real area-wide truth in the TP network is weaker than that in the MP area. For instance, the unavailability of soil moisture measurements in the wetlands of the TP network would lead to underestimation of soil moisture by SA. But, the ATI upscaling exploits satellite high-resolution thermal information to reflect soil moisture spatial variability, and thus alleviates the problem of lack of representativeness of a limited number of stations.

The BK method (Fig. 3a3 and b3) effectively reduces both ubRMSD and RMSD compared to the SA method in the TP network, but it seems not to work well in the MP network area (Table 1). The BK is a geostatistically based method, with the semivariogram among moisture stations as the extra information. Fig. 5 indicates the typical spherical semivariogram for the two network areas. The 
range of the theoretical semivariogram model characterizes the correlation length of soil moisture in space. As can be seen, the range in the TP area is almost three times higher than in the MP area, explaining why the BK method works in the TP. Nevertheless, the upscaling performance of the ATI method is superior to that of the BK approach for both networks. As well known, the nugget of the theoretical semivariogram describes spatial sources of variation at distances smaller than the sampling interval. In the TP network area, the separation distance among the stations used in this study generally exceeds $5 \mathrm{~km}$, implying that the moisture information under this distance cannot be captured by the semivariogram and thus cannot be exploited by the BK upscaling approach. By contrast, the ATI upscaling method is able to detect this information by using 1-km MODIS LST products.

The MB upscaled soil moisture (Fig. 3a4 and b4) in this study is based on the GLDAS Noah 0.25-degree soil moisture product due to its popularity. Table 1 also shows that the performance of the MB upscaling approach is rather unstable. Its performance is inferior to those of both the ATI and BK methods except the ubRMSD in the SMOS assimilation case. Its instability is due to the fact that the model-simulated soil moisture depends on many factors such as atmospheric forcing and model parameters. It is difficult to accurately determine these factors at a fine spatial resolution. So, uncertainties in spatial variability of the simulated soil moisture are large and thus the quality of the MB upscaled soil moisture cannot be guaranteed, at least in the two networks of concern. However, as pointed out by Crow et al. (2005), the MB upscaling method is reliable when the factors impacting soil moisture simulation can be available at a fine spatial resolution.

\section{Conclusions}

Many satellite missions have been initiated for mapping soil moisture globally. The estimated soil moisture should be evaluated against the ground truth before applied. Therefore, a number of soil moisture networks are established around the world and each of them has tens of moisture sensors so as to represent the spatial variability of soil moisture. Then, an upscaling method is implemented to obtain footprint-averaged soil moisture as the ground truth. In this study, four upscaling methods are implemented in two networks located on the Tibetan Plateau and the Mongolian Plateau, respectively. Due to unavailability of the true area-wide soil moisture, the performance of the four upscaling methods is inter-compared according to their stability against soil moisture estimates from assimilating satellite observations. A caveat must be realized that uncertainties in the assimilation soil moisture might impact the values of the performance metrics. Even so, the following conclusions can still be drawn with confidence not only because they are based on the analyses for different satellite data and for distinct areas, but also because they are supported by the theoretical grounds behind the upscaling methods.

The model-based upscaling approach is found to be the most unstable because it is difficult to determine crucial factors for soil moisture modeling at a fine spatial resolution on a relatively large area. Certainly, once these factors such as atmospheric forcing and soil texture can be accurately quantified, its effectiveness is prospective. The block kriging upscaling method performs not worse than the simple averaging method. If the correlation length between station data is comparable to the size of the satellite footprint, this method can generate more representative upscaled soil moisture than the simple averaging method, but it is unable to exploit soil moisture information hiding in the gaps between two stations. The ATI-based upscaling approach can overcome this disadvantage by introducing fine-resolution satellite thermal signals, and its performance proves to be the most stable among the four upscaling methods.
It is noted that all above analyses and conclusions are made based on about four months of data over the Tibetan Plateau and the Mongolian Plateau and, thus, are not yet the definitive answer to the question of which is the best upscaling method. So, more investigations are needed to evaluate the performance of these four upscaling methods in other regions with distinct climates and land covers. Nevertheless, this study demonstrates that it is critical to select a suitable upscaling approach for the effective evaluation of satellite-based soil moisture. Otherwise, the uncertainties hiding in the upscaling method will be incorrectly attributed to errors in the satellite soil moisture products, and thus mislead us when implementing them into practice.

\section{Acknowledgements}

This work was supported by the Natural Science Foundation of China (Grant Nos. 41171268 and, China "863" project under contract 2013AA122801, and the CMA Special Fund for Scientific Research in the Public Interest (Grant No. GYHY201206008).

\section{Appendix A}

A land surface assimilation framework for assimilating microwave satellite signals is primarily composed of three components: a land surface model as the model operator to simulate state variables (temperature and soil moisture), a radiative transfer equation as the observation operator linking the model state variables and the satellite signals, and an assimilation algorithm to merge the model and the satellite observations to optimally estimate and update the model state.

In the assimilation framework developed by Yang et al. (2007), the simple biosphere model ( $\mathrm{SiB} 2$ ) is chosen as the model operator, including one canopy layer and three soil layers. It is a typical dualsource model parameterizing heat transfer from both the canopy and the ground. A two-stream scheme is used to compute surface albedo, a photosynthesis-based stomatal resistance model is used to simulate the exchange of carbon dioxide and water vapor between the vegetation and the atmosphere, and an aerodynamic canopy transfer scheme is used to describe heat transfer from both the canopy and the ground.

As mentioned in the main body, both microwave brightness temperatures and satellite-based soil moisture can be assimilated. When brightness temperatures are assimilated, a zero-order microwave radiative transfer model is applied to link them with model state variables (soil moisture and temperature) as follows:

$$
\begin{aligned}
T_{\mathrm{bp}}= & T_{g}\left(1-\Gamma_{p}\right) \exp \left(-\tau_{c}\right)+T_{c}(1-\omega)\left[1-\exp \left(-\tau_{c}\right)\right]\left[1+\Gamma_{p}\right. \\
& \left.\times \exp \left(-\tau_{c}\right)\right],
\end{aligned}
$$

where the subscript $p$ denotes the vertical or horizontal polarization, $T_{\mathrm{bp}}(K)$ the simulated brightness temperature, $\Gamma_{p}$ the soil reflectivity, $\tau_{c}$ the vegetation optical depth, and $\omega$ the vegetation single scattering albedo. A $Q-h$ model is used to calculate the soil reflectivity as follows:

$\Gamma_{p}=\left[(1-Q) R_{p}+Q R_{q}\right] \exp (-h)$,

where $Q$ and $h$ are empirical surface roughness parameters, and $R$ the Fresnel power reflectivity with a smooth soil surface, which is determined using the following equations:

$$
R_{h}=\left|\frac{\cos \gamma-\sqrt{\varepsilon_{r}-\sin ^{2} \gamma}}{\cos \gamma+\sqrt{\varepsilon_{r}-\sin ^{2} \gamma}}\right|^{2},
$$

and 
$R_{v}=\left|\frac{\varepsilon_{r} \cos \gamma-\sqrt{\varepsilon_{r}-\sin ^{2} \gamma}}{\varepsilon_{r} \cos \gamma+\sqrt{\varepsilon_{r}-\sin ^{2} \gamma}}\right|^{2}$,

where $h$ and $v$ denote the horizontal and vertical polarization, $\gamma$ the incident angle, and $\varepsilon_{r}$ the soil dielectric constant. The soil dielectric constant is computed as follows:

$\varepsilon_{r}=\left[1+\left(1-\theta_{\mathrm{sat}}\right)\left(\varepsilon_{s}^{a}-1\right)+\theta_{s}^{b} \varepsilon_{\mathrm{fw}}^{a}-\theta_{s}\right]^{1 / a}$,

where $\theta_{\text {sat }}$ denotes the porosity, $\theta_{s}$ the surface soil moisture, $\varepsilon_{s}=4.7+0.0 j$ the dielectric constant for mineral soil, $\varepsilon_{\mathrm{fw}}$ the dielectric constant of free water, $a=0.65$, and $b$ the coefficient dependent upon the soil texture. The parameters in Eqs. (A1) and (A2) are dependent on wave frequency and can be parameterized as follows:

$h=(k \cdot s)^{\sqrt{0.1 \cos \gamma}}$,
$Q=Q_{0}(k \cdot s)^{0.795}$,
$\tau_{c}=\frac{b^{\prime}(100 \lambda)^{\chi} w_{c}}{\cos \gamma}$,
$w_{c}=\exp (L A I / 3.3)-1$,

(anclo

and

$\omega=\frac{0.00083}{\lambda}$,

where $\lambda(\mathrm{m})$ is the wavelength, $k$ the wave number defined as $2 \pi / \lambda$, $s$ the standard deviation of surface roughness, $w_{c}\left(\mathrm{~kg} \mathrm{~m}^{-2}\right)$ the vegetation water content, and $Q_{0}, b^{\prime}$, and $\chi$ the empirical coefficients.

Here, some specifics are given to the process of assimilating AMSR-E brightness temperatures. As for assimilating SMOS retrieved soil moisture, a similar procedure is taken. The assimilation consists of two passes, both of which assimilate observed brightness temperatures of the vertical polarization at a lower frequency $(6.9 \mathrm{GHz})$ and a higher frequency $(18.7 \mathrm{GHz})$ due to its relative insensitivity to vegetation coverage (Fujii, 2005). The lower frequency $T_{b}$ is much more sensitive to near-surface soil moisture than the higher one, and thus their difference is correlated with soil wetness (Koike et al. 2000). A soil wetness index (SWI) is then defined by SWI $=2\left(T_{\mathrm{bv}}^{18.7}-T_{\mathrm{bv}}^{6.9}\right) /\left(T_{\mathrm{bv}}^{18.7}+T_{\mathrm{bv}}^{6.9}\right)$. A high SWI value corresponds to a wet surface, and a low SWI value corresponds to a dry surface.

On the one hand, driving Eq. (A1) needs many inputs, including surface soil water content $\left(\theta_{s}\right)$, ground temperature $\left(T_{g}\right)$, canopy temperature $\left(T_{c}\right)$, vegetation water content $\left(w_{c}\right)$, canopy parameters, surface roughness parameters, and soil texture. The simulation of surface variables by SiB2 also needs a number of soil and vegetation parameters. The modeled $T_{b}$ is thus sensitive to some parameters in SiB2 and the microwave radiative transfer. In the first pass, these parameters are obtained through minimizing a cost function that accounts for the difference between modeled and observed brightness temperatures during a long-term $\left(t_{\mathrm{pass} 1}-\right.$ $\sim$ months). The cost function includes an observation error term and a background error term. The observation error term is defined by:

$F_{\mathrm{obs}}=\sum_{t=0}^{t_{\mathrm{pass} 1}}\left[\left(T_{\mathrm{bv}, \mathrm{est}}^{6.9}-T_{\mathrm{bv}, \mathrm{obs}}^{6.9}\right)^{2}+\left(T_{\mathrm{bv}, \mathrm{est}}^{18.7}-T_{\mathrm{bv}, \mathrm{obs}}^{18.7}\right)^{2}\right]$,

where the subscript obs denotes observed value, est is the modeled value.

In the first pass, the background error term is not directly accounted for in the cost function. Instead, it is realized through an adjustment of surface soil water content $\left(\theta_{s}\right)$ at each observing time so that the renewed SWI value, which depends on $\theta_{s}$, is close to $\left(\mathrm{SWI}_{\mathrm{est}}+\mathrm{SWI}_{\mathrm{obs}}\right) / 2$. Note that this adjustment is implemented after (rather than before) adding the bias term $\left(T_{\mathrm{bv}, \text { est }}-T_{\mathrm{bv}, \mathrm{obs}}\right)^{2}$ into Eq. (A11). In addition, because of model deficiencies and errors in forcing data, simulated soil moisture without this adjustment may become unrealistic, which then results in no correlation between $T_{\mathrm{bv}, \mathrm{est}}$ and $T_{\mathrm{bv}, \mathrm{obs}}$ no matter how to tune parameter values. Therefore, this adjustment is critical for the parameter optimization.

The optimal parameter values are then transferred into the second pass for retrieving soil moisture and surface energy budget by assimilating the brightness temperature into SiB2. This pass only optimizes the near-surface soil moisture, and its assimilation window ( $t_{\text {pass2 }} \sim 1$ day) is much shorter than that for the first pass. Its cost function is defined by

$$
\begin{aligned}
F_{\mathrm{obs}}= & \sum_{t=0}^{t_{\text {pass } 2}}\left[\left(T_{\mathrm{bv}, \mathrm{est}}^{6.9}-T_{\mathrm{bv}, \mathrm{obs}}^{6.9}\right)^{2}+\left(T_{\mathrm{bv}, \mathrm{est}}^{18.7}-T_{\mathrm{bv}, \mathrm{obs}}^{18.7}\right)^{2}\right] \\
& +\left[\left(T_{\mathrm{bv} 0, \mathrm{bg}}^{6.9}-T_{\mathrm{bv0} 0}^{6.9}\right)^{2}+\left(T_{\mathrm{bv} 0, \mathrm{bg}}^{18.7}-T_{\mathrm{bv0}}^{18.7}\right)^{2}\right]
\end{aligned}
$$

where $T_{\mathrm{bv} 0, \mathrm{bvg}}$ and $T_{\mathrm{bv0}}$ are the simulated brightness temperature at the initial time of each assimilation cycle using the background value of $\theta_{s, 0}$ (i.e. $\theta_{s, \text { bg }}$ ) and using a renewed $\theta_{s, 0}$ value, respectively. It is noted that the first pass only needs execution once, because the optimized parameters only include static model parameters and initial soil water conditions. It can be implemented with previous data before real-time assimilation of satellite data in the second pass.

\section{References}

Al Bitar, A. et al., 2012. Evaluation of SMOS soil moisture products over continental US using the SCAN/SNOTEL network. IEEE T Geosci. Remote 50 (5), 1572-1586. Bloschl, G., Grayson, R., 2001. In: Grayson, R., Bloschl, G. (Eds.), Spatial Observations and Interpolation. Spatial Patterns in Catchment Hydrology: Observations and Modelling. Cambridge University Press, UK, pp. 17-50, ISBN 0-521-63316-8.

Chen, Y.Y. et al., 2011. Improving land surface temperature modeling for dry land of China. J. Geophys. Res. - Atmospheres 116, D20104.

Crow, W.T., Wood, E.F., 2003. The assimilation of remotely sensed soil brightness temperature imagery into a land surface model using Ensemble Kalman filtering: a case study based on ESTAR measurements during SGP97. Adv. Water Resour. 26 (2), 137-149.

Crow, W.T., Ryu, D., Famiglietti, J.S., 2005. Upscaling of field-scale soil moisture measurements using distributed land surface modeling. Adv. Water Resour. 28 (1) $1-14$.

Crow, W.T. et al., 2012. Upscaling sparse ground-based soil moisture observations for the validation of coarse-resolution satellite soil moisture products. Rev. Geophys. 50 (2), RG2002.

Das, N.N., Entekhabi, D., Njoku, E.G., 2011. An algorithm for merging SMAP radiometer and radar data for high-resolution soil-moisture retrieval. IEEE T Geosci. Remote 49 (5), 1504-1512.

Entekhabi, D. et al., 2010a. The Soil Moisture Active Passive (SMAP) mission. Proc. IEEE 98 (5), 704-716.

Entekhabi, D., Reichle, R.H., Koster, R.D., Crow, W.T., 2010b. Performance metrics for soil moisture retrievals and application requirements. J. Hydrometeorol. 11 (3), $832-840$.

Fujii, H., 2005. Development of a microwave radiative transfer model for vegetated land surface based on comprehensive in-situ observations. PhD Thesis. The University of Tokyo.

Hirabayashi, Y., Kanae, S., Struthers, I., Oki, T., 2005. A 100-year (1901-2000) global retrospective estimation of the terrestrial water cycle. J. Geophys. Res. 110, D19101. http://dx.doi.org/10.1029/2004JD005492.

Jackson, T.J. et al., 1999. Soil moisture mapping at regional scales using microwave radiometry: the southern great plains hydrology experiment. IEEE T Geosci. Remote 37 (5), 2136-2151.

Jackson, T.J. et al., 2010. Validation of Advanced Microwave Scanning Radiometer soil moisture products. IEEE T Geosci. Remote 48 (12), 4256-4272.

Jackson, T.J. et al., 2012. Validation of Soil Moisture and Ocean Salinity (SMOS) soil moisture over watershed networks in the U.S. IEEE T Geosci. Remote 50 (5), 1530-1543.

Kaihotsu, I., Yamanaka, T., Koike, T., Oyunbaatar, D., Davaa, G., 2005. Ground truth for evaluation of soil moisture and geophysical/vegetation parameters related to ground surface conditions with AMSR and GLI in the Mongolian Plateau, ground-based observations for the ADEOS II/AQUA validation in the Mongolian Plateau. Jpn. Aerospace Explor. Agency, 5-21.

Kerr, Y.H. et al., 2010. The SMOS mission: new tool for monitoring key elements of the global water cycle. Proc. IEEE 98 (5), 666-687.

Kerr, Y.H. et al., 2012. The SMOS soil moisture retrieval algorithm. IEEE T Geosci. Remote 50 (5), 1384-1403. 
Koike, T., Shimo, C., Ohta, T., Fujii, H., Shibata, A., 2000. Development and validation of a microwave radiometer algorithm for land surface hydrology. Ann. J. Hydraulic Eng. JSCE 44, 247-252.

Koster, R.D., Mahanama, S.P.P., Livneh, B., Lettenmaier, D.P., Reichle, R.H., 2010. Skill in streamflow forecasts derived from large-scale estimates of soil moisture and snow. Nat. Geosci. 3 (9), 613-616.

Lu, H. et al., 2012. Improving land surface soil moisture and energy flux simulations over the Tibetan Plateau by the assimilation of the microwave remote sensing data and the GCM output into a land surface model. Int. J. Appl. Earth Obs. Geoinf. 17, 43-54

Merlin, O., Walker, J.P., Chehbouni, A., Kerr, Y., 2008. Towards deterministic downscaling of SMOS soil moisture using MODIS derived soil evaporative efficiency. Remote Sens. Environ. 112 (10), 3935-3946.

Montzka, C. et al., 2011. Hydraulic parameter estimation by remotely-sensed top soil moisture observations with the particle filter. J. Hydrol. 399 (3-4), 410-421.

Njoku, E.G., Jackson, T.J., Lakshmi, V., Chan, T.K., Nghiem, S.V., 2003. Soil moisture retrieval from AMSR-E. IEEE T Geosci. Remote 41 (2), 215-229.

Pan, M., Wood, E.F., 2006. Data assimilation for estimating the terrestrial water budget using a constrained ensemble Kalman filter. J. Hydrometeorol. 7 (3), 534-547.

Piles, M. et al., 2011. Downscaling SMOS-derived soil moisture using MODIS visible/ infrared data. Geosci. Remote Sensing, IEEE Trans. 49 (9), 3156-3166.

Qin, J. et al., 2009. Simultaneous estimation of both soil moisture and model parameters using particle filtering method through the assimilation of microwave signal. J. Geophys. Res. 114 (D15), D15103.

Qin, J. et al., 2013. Spatial upscaling of in-situ soil moisture measurements based on MODIS-derived apparent thermal inertia. Remote Sens. Environ. 138, 1-9.
Reichle, R.H., McLaughlin, D.B., Entekhabi, D., 2002. Hydrologic data assimilation with the ensemble Kalman filter. Mon. Weather Rev. 130 (1), 103-114.

Rodell, M. et al., 2004. The Global Land Data Assimilation System. B Am. Meteorol. Soc. 85 (3), 381-394.

Sanchez, N., Martinez-Fernandez, J., Scaini, A., Perez-Gutierrez, C., 2012. Validation of the SMOS L2 soil moisture data in the REMEDHUS network (Spain). IEEE T Geosci. Remote 50 (5), 1602-1611.

Sellers, P.J. et al., 1996. A revised land surface parameterization (SiB2) for atmospheric GCMS. Part I: Model formulation. J. Climate 9 (4), 676-705.

Seneviratne, S.I. et al., 2010. Investigating soil moisture-climate interactions in a changing climate: a review. Earth - Sci. Rev. 99 (3-4), 125-161.

Sheffield, J. Wood, E.F. 2007. Characteristics of global and regional drought, 1950 2000: analysis of soil moisture data from off-line simulation of the terrestrial hydrologic cycle. J. Geophys. Res. 112, D17115.

Vinnikov, K.Y., Robock, A., Qiu, S., Entin, J.K., 1999. Optimal design of surface networks for observation of soil moisture. J. Geophys. Res. 104 (D16), 19743 19749.

Yang, K. et al., 2007. Auto-calibration system developed to assimilate AMSR-E data into a land surface model for estimating soil moisture and the surface energy budget. J. Meteorol. Soc. Jpn 85, 229-242.

Yang, K. et al., 2013. A multiscale soil moisture and freeze-thaw monitoring network on the third pole. B Am. Meteorol. Soc. 94 (12), 1907-1916.

Zhao, L. et al., 2014. The scale-dependence of SMOS soil moisture accuracy and its improvement through land data assimilation in the central Tibetan Plateau. Remote Sens. Environ. 152, 345-355. 